PROCEEDINGS OF THE

AMERICAN MATHEMATICAL SOCIETY

Volume 136, Number 11, November 2008, Pages 3931-3940

S 0002-9939(08)09602-0

Article electronically published on June 24, 2008

\title{
SPECIFICATION PROPERTY AND DISTRIBUTIONAL CHAOS ALMOST EVERYWHERE
}

\author{
PIOTR OPROCHA AND MARTA ŠTEFÁNKOVÁ
}

(Communicated by Jane M. Hawkins)

\begin{abstract}
Our main result shows that a continuous map $f$ acting on a compact metric space $(X, \rho)$ with a weaker form of specification property and with a pair of distal points is distributionally chaotic in a very strong sense. Strictly speaking, there is a distributionally scrambled set $S$ dense in $X$ which is the union of disjoint sets homeomorphic to Cantor sets so that, for any two distinct points $u, v \in S$, the upper distribution function is identically 1 and the lower distribution function is zero at some $\varepsilon>0$. As a consequence, we describe a class of maps with a scrambled set of full Lebesgue measure in the case when $X$ is the $k$-dimensional cube $I^{k}$. If $X=I$, then we can even construct scrambled sets whose complements have zero Hausdorff dimension.
\end{abstract}

\section{INTRODUCTION}

In 1981 Gy. Targonski formulated a question during the International Symposium in France as to whether there exists a continuous map $f$ of the interval $I$, chaotic in the sense of $\mathrm{Li}$ and Yorke, whose scrambled set has positive Lebesgue measure (denoted by $\mathcal{L}(\cdot)$ ). In other words, is there an $f \in \mathcal{C}(I)$ and a set $S \subset I$ with $\mathcal{L}(S)>0$ such that, for any distinct $u, v$ in $S$, $\liminf _{n \rightarrow \infty}\left|f^{n}(u)-f^{n}(v)\right|=0$ and $\lim \sup _{n \rightarrow \infty}\left|f^{n}(u)-f^{n}(v)\right|>0$ ? In 1983 Smítal 18 proved that the tent map given by $f(x)=1-|2 x-1|, 0 \leq x \leq 1$, has scrambled set of full outer Lebesgue measure but no scrambled set of positive measure; the notion of scrambled set was used for the first time in this paper. In 1984 Kan [11] and Smítal [19] proved that a scrambled set can have positive measure. Misiurewicz in 1985 gave an example of chaos (in the sense of Li and Yorke) almost everywhere 14. A slightly stronger result, but with an easier proof, was given by Bruckner and $\mathrm{Hu}$ [7 in 1987; chaos in this paper is extremal, that is, $\limsup _{n \rightarrow \infty}\left|f^{n}(u)-f^{n}(v)\right|=1$ for any $u \neq v$ in the scrambled set. This construction was further improved and simplified in 2000 by Babilonová [1. All these results concern chaos in the sense of $\mathrm{Li}$ and Yorke. One of the most important extensions of the concept of chaos in the sense of $\mathrm{Li}$ and Yorke is distributional chaos as introduced in 16. In 2003, a general method for the construction of interval maps distributionally chaotic almost everywhere (see the definition in the next section) was obtained [2]. Let us also note that a particular example of an interval map distributionally chaotic almost everywhere was rediscovered in 2007 [13]. There are also recent papers concerning

Received by the editors September 27, 2007.

2000 Mathematics Subject Classification. Primary 37B05; Secondary 54H20.

(C)2008 American Mathematical Society

Reverts to public domain 28 years from publication 
the topological size of Li-Yorke scrambled sets for continuous maps of a compact metric space; see, e.g., 10] or [4. But there are no results concerning topological size of distributionally scrambled sets.

In the present paper we essentially improve the above quoted results in two directions. First, we show that similar results concerning distributional chaos can be proved for continuous maps of the $k$-dimensional unit cube $I^{k}$. For one-dimensional maps we show, among others, that distributional chaos can exist everywhere except for a set of zero Hausdorff dimension. Our main tool is Theorem 2, which gives a sufficient condition for a continuous map of a compact metric space $(X, \rho)$ to have a distributionally scrambled set which is a $c$-dense $F_{\sigma}$ subset of $X$.

\section{TERminOLOGY}

Let $(X, \rho)$ be a compact metric space. Let us denote by $C(X)$ the set of continuous self-maps acting on $X$. Let $f \in C(X)$. By $\omega_{f}(x)$ we denote the omega-limit set of a point $x \in X$, that is, the set of limit points of the orbit of $x$ under the action of $f$. Point $x$ is said to be periodic if $f^{n}(x)=x$ for some $n \geq 1$. We denote by $\operatorname{Per}(f)$ the set of periodic points of $f$.

By $I$ we denote the compact unit interval $[0,1]$. By the $k$-dimensional unit cube we mean the set $I^{k}$, where $k \geq 1$. The Lebesgue measure on $I^{k}$ will be denoted by $\mathcal{L}$. By a perfect set we mean a compact set without isolated points. By a Cantor set we mean a nonempty, perfect and totally disconnected set.

2.1. Specification properties. The specification property was introduced by Bowen in [6] (see [17] or [8] for further examples of maps with the specification property and for their basic properties). We recall the definition below; however, we will use the terminology (the names of properties) introduced in 3 because with this terminology it is easier to distinguish between different kinds of specification properties.

Definition 1. A continuous function $f$ acting on a nondegenerate (i.e., with at least two elements) compact metric space $(X, \rho)$ has the strong specification property (briefly $S S P$ ) if, for any $\delta>0$, there is a positive integer $N_{\delta}$ such that for any integer $s \geq 2$, any set $\left\{y_{1}, \ldots, y_{s}\right\}$ of $s$ points of $X$, and any sequence $0=j_{1} \leq k_{1}<j_{2} \leq$ $k_{2}<\cdots<j_{s} \leq k_{s}$ of $2 s$ integers with $j_{m+1}-k_{m} \geq N_{\delta}$ for $m=1, \ldots, s-1$, there is a point $x$ in $X$ such that, for each positive integer $m \leq s$ and all integers $i$ with $j_{m} \leq i \leq k_{m}$, the following two conditions hold:

$$
\begin{gathered}
\rho\left(f^{i}(x), f^{i}\left(y_{m}\right)\right)<\delta, \\
f^{n}(x)=x, \quad \text { where } n=N_{\delta}+k_{s} .
\end{gathered}
$$

If $f$ fulfills the above-mentioned conditions (11) and (2) only for the special case $s=2$, then we say that $f$ has periodic specification property (briefly PSP). If additionally periodicity condition (2) is omitted, we say that $f$ fulfills the weak specification property.

To prove the main results of this paper we need only the weak specification property. We will reformulate it in the following (equivalent) way.

Definition 2. A continuous map $f$ of a compact metric space $(X, \rho)$ has the weak specification property, briefly $W S P$, if for any $\eta>0$ and any $n$ there is a $K=K(\eta, n)$ such that for any sequence of integers $T_{0}=0<T_{1}<T_{2}<\cdots<T_{n}$ such that 
$K<T_{i+1}-T_{i}$, whenever $1 \leq i<n$, and any $u, v \in X$, there is a point $z \in X$ such that

$$
\rho\left(f^{j}(z), f^{j}(u)\right)<\eta \text { if } T_{2 i} \leq j \leq T_{2 i+1}-K \text { and } 2 i+1 \leq n,
$$

and

$$
\rho\left(f^{j}(z), f^{j}(v)\right)<\eta \text { if } T_{2 i-1} \leq j \leq T_{2 i}-K \text { whenever } 2 i \leq n .
$$

2.2. Distributional chaos. Let $f \in C(X)$, where $(X, \rho)$ is a compact metric space. For any positive integer $n$, points $x, y \in X$ and $t \in \mathbb{R}$ let

$$
\Phi_{x y}^{(n)}(t)=\frac{1}{n}\left|\left\{i: \rho\left(f^{i}(x), f^{i}(y)\right)<t, 0 \leq i<n\right\}\right|,
$$

where $|A|$ denotes the cardinality of the set $A$. Let us denote by $\Phi_{x y}$ and $\Phi_{x y}^{*}$ the following functions:

$$
\Phi_{x y}(t)=\liminf _{n \rightarrow \infty} \Phi_{x y}^{(n)}(t), \quad \Phi_{x y}^{*}(t)=\limsup _{n \rightarrow \infty} \Phi_{x y}^{(n)}(t) .
$$

Both functions $\Phi_{x y}$ and $\Phi_{x y}^{*}$ are nondecreasing, $\Phi_{x y}(t)=\Phi_{x y}^{*}(t)=0$ for $t<0$ and $\Phi_{x y}(t)=\Phi_{x y}^{*}(t)=1$ for $t>\operatorname{diam} X$.

Remark 1. Should we later need to emphasise that $\Phi_{x y}(t)$ (and $\left.\Phi_{x y}^{*}(t)\right)$ are based on the function $f$, we will explicitly use the notation $\Phi_{x y}(f, t)$ (and $\Phi_{x y}^{*}(f, t)$ ).

Definition 3. A pair of points $(x, y) \in X \times X$ is called distributionally chaotic of type 1 if

$$
\begin{aligned}
& \Phi_{x y}(s)=0 \text { for some } s>0 \text { and } \\
& \Phi_{x y}^{*}(t)=1 \text { for all } t>0 .
\end{aligned}
$$

A set containing at least two points is called a distributionally scrambled set of type 1 ( $D 1$-scrambled, for short) if any pair of its distinct points is distributionally chaotic of type 1.

A map $f$ is distributionally chaotic of type 1 (DC1, for short) if it has an uncountable $D 1$-scrambled set $S$. We say that distributional chaos of type 1 is uniform if there exists $T>0$ such that $\Phi_{x y}(T)=0$ for any distinct $x, y \in S$.

Definition 4. We say that a map $f \in C(X)$ exhibits extremal distributional chaos if it exhibits distributional chaos of type 1 with a $D 1$-scrambled set $S$ such that $\Phi_{x y}(s)=0$ for any distinct points $x, y \in S$ and any $s<\operatorname{diam} X$.

Definition 5. We say that a continuous map from the unit cube $I^{k}$ into itself displays (uniform) distributional chaos of type 1 almost everywhere ((uniform) DC1 a.e., for short) if there exists a distributionally scrambled set $S \subset I^{k}$ of type 1 such that $\mathcal{L}(S)=1$ (and distributional chaos is uniform, respectively).

\section{Specification PROPERTy AND Distributional ChaOS}

In this section we will construct $D 1$-scrambled sets which are homeomorphic to sets with full Lebesgue measure. An appropriate homeomorphism is obtained by a direct application of the following theorem by Oxtoby and Ulam (see [15, Thm. 9]).

Theorem 1 (Oxtoby and Ulam). Let $B \subset I^{k}$ and suppose that there exists a sequence $\left\{S_{n}\right\}_{n=1}^{\infty}$ of perfect sets $S_{n} \subset B$ such that $\bigcup_{n \in \mathbb{N}} S_{n}$ is dense in $I^{k}$. Then there exists a homeomorphism $h: I^{k} \rightarrow I^{k}$ such that $\left.h\right|_{\partial I^{k}}=i d$ and $\mathcal{L}(h(B))=1$. 
It should be noted that twenty-five years later a much weaker version of this result, for $k=1$, was rediscovered in [9]. The following theorem is the main result of the paper. It is used as background for further theorems and examples.

Theorem 2. Let $f$ be a continuous map of a compact metric space $(X, \rho)$ with WSP such that $X$ contains a pair of distal points $u, v$ (i.e. $\liminf _{n \rightarrow \infty} \rho\left(f^{n}(x), f^{n}(y)\right)>$ $0)$. Then $f$ is DC1 and possesses a dense D1-scrambled set $S$ which is an at most countable sum of pairwise disjoint Cantor sets and such that, for any distinct $x, y$ in $S$,

$$
\Phi_{x y}^{*} \equiv 1 \text { and } \Phi_{x y}(\varepsilon)=0 \text {, where } \varepsilon=\liminf _{n \rightarrow \infty} \rho\left(f^{n}(u), f^{n}(v)\right) .
$$

To prove this theorem we need the following two lemmas. For any $\alpha$ in the set $\{u, v\}^{n}$ of all ordered $n$-tuples of two symbols, let $B_{\alpha} \subset X$ be a nonempty closed set with diameter less than $d(n)$, with $\lim _{n \rightarrow \infty} d(n)=0$, such that

$$
B_{\alpha} \cap B_{\beta}=\emptyset \text { and } B_{\alpha u} \cup B_{\alpha v} \subset B_{\alpha} \text {, if } \alpha, \beta \in\{u, v\}^{n}, \alpha \neq \beta \text {, }
$$

where $\alpha u$ denotes the $(n+1)$-tuple $\alpha_{1} \alpha_{2} \ldots \alpha_{n} u \in\{u, v\}^{n+1}$ (and similarly for $\alpha v$ ). The following result is obvious (note that much stronger results of this kind are well known [12, e.g. the Alexandroff-Hausdorff theorem).

Lemma 1. The set $\bigcap_{n=1}^{\infty} \bigcup_{\alpha \in\{u, v\}^{n}} B_{\alpha}$ is homeomorphic to the middle-third Cantor set $C \subset I$.

We fix the following notation. Let $\delta>0$ (it will be specified later), and let $\delta_{i}=2^{-i} \delta$, for any $i \geq 1$. Let $K_{i}:=K\left(\delta_{i}, 4^{i}\right)$, for $i \geq 1$ (cf. definition of WSP). Let $0=T_{0}<T_{1}<T_{2}<\cdots$ be an increasing sequence of integers such that $K_{i}<T_{i}-T_{i-1}$, for any $i \geq 1$, and

$$
\lim _{i \rightarrow \infty} \frac{T_{i+1}-K_{i+1}}{T_{i}}=\infty .
$$

Finally, if $\rho\left(f^{j}(x), f^{j}(y)\right)<\eta$ for any $j$ with $a \leq j \leq b-K(\eta, n)$, where $K(\eta, n)$ is given by WSP, then we say that $x \eta$-traces $y$ on the interval $J=[a, b]$. Thus, e.g., (3) means that $z \eta$-traces $u$ on any $\left[T_{2 i}, T_{2 i+1}\right]$.

Lemma 2. Let $X, f$, and $u, v$ be as in Theorem 2, Let $\nu=\{n(i)\}_{i=1}^{\infty}$ be an increasing sequence of positive integers, and let $G \subset X$ be an open ball with center $g$ and radius $\delta$. Then there is an infinite subsequence $\mu=\{m(i)\}_{i=1}^{\infty}$ of $\nu$ and a Cantor set $S \subset G$ such that (77) is valid for any $x \neq y$ in $S$. Moreover, any $x \in S \delta_{i}$-traces $u$ on any interval $\left[T_{m(i)}, T_{m(i)+1}\right]$ if $i$ is odd, and $\delta_{i}$-traces $v$ on $\left[T_{m(i)}, T_{m(i)+1}\right]$ otherwise.

Proof. The proof is a little bit technical, so we first describe the idea behind it. We use WSP to approximate the desired Cantor set in an inductive construction (similar to the construction of the Cantor ternary set). We define a subsequence $\mu=\{m(i)\}_{i=1}^{\infty}$ so that the number of iterations needed for a change of a traced orbit (applying WSP) is very small when compared to $\left|T_{m(i)+1}-T_{m(i)}\right|$. We also note that the definition of WSP (Definition 2) can be easily reformulated using any finite sequence of points $u_{1}, \ldots, u_{k}$ instead of just a pair of two points $u, v$.

We now turn to the construction. Since $f$ has WSP, there are members $m(1)<$ $m(2)$ in $\nu$ greater than 1 and points $x_{u}, x_{v} \in G$ which $\delta_{2}$-trace $g$ on $\left[0, T_{1}\right], \delta_{2}$-trace $u$ on $\left[T_{m(1)}, T_{m(1)+1}\right]$, and $\delta_{2}$-trace $v$ on $\left[T_{m(2)}, T_{m(2)+1}\right]$. Moreover, these points can be chosen in such a way that $x_{t} \delta_{2}$-traces $t$ on $\left[T_{m(2)+1}, T_{m(2)+2}\right]$, for $t \in\{u, v\}$. 
By continuity, there are disjoint compact balls $B_{u}, B_{v} \subset G$ such that any point $x \in B_{t} \delta_{3}$-traces $x_{t}$ on $\left[0, T_{m(2)+2}\right]$, for $t=u, v$. Without loss of generality we can assume that the diameter of either $B_{u}$ or $B_{v}$ is $\delta_{j(1)}$ where $j(1) \geq 3$.

Again by the WSP there are members $m(3), m(4)$ in $\nu$ such that $m(3)>m(2)+2$, $m(4)>m(3)+3$, and points $x_{u u}, x_{u v}$ interior to $B_{u}$, and $x_{v u}, x_{v v}$ interior to $B_{v}$ that $\delta_{j(1)}$-trace $u$ on $\left[T_{m(3)}, T_{m(3)+1}\right], \delta_{j(1)}$-trace $v$ on $\left[T_{m(4)}, T_{m(4)+1}\right]$. Additionally, we may assume that

$$
\begin{aligned}
& x_{u u}, x_{u v} \delta_{j(1)} \text {-trace } u \text {, and } x_{v u}, x_{v v} \delta_{j(1)} \text {-trace } v \text { on }\left[T_{m(4)+1}, T_{m(4)+2}\right] \\
& x_{u u}, x_{v u} \delta_{j(1)} \text {-trace } u \text {, and } x_{u v}, x_{v v} \delta_{j(1)} \text {-trace } v \text { on }\left[T_{m(4)+2}, T_{m(4)+3}\right] .
\end{aligned}
$$

To finish the second step, similarly as before we find disjoint compact sets $B_{u u}, B_{u v}$ in the interior of $B_{u}$ and $B_{v u}, B_{v v}$ in the interior of $B_{v}$ such that any point $x \in B_{\alpha}$ $\delta_{j(1)+1}$-traces $x_{\alpha}$ on $\left[0, T_{m(4)+3}\right]$, for any $\alpha \in\{u, v\}^{2}$. Without loss of generality we can assume that the diameter of any $B_{\alpha}$ is $\delta_{j(2)}$, with $j(2)>j(1)+1$.

By induction, for any $k \in \mathbb{N}$ and any $\alpha \in\{u, v\}^{k}$ there is a compact ball $B_{\alpha}$, integers $m(1)<m(2)<\cdots<m(2 k)$ from $\nu$, and positive integers $j(1)<j(2)<$ $\ldots<j(k)$ such that if $x, y$ are distinct points of $B_{\alpha}$, then $x \delta_{j(k-1)}$-traces $y$ on $\left[0, T_{m(2 k)+k}\right]$. The balls $B_{\alpha}$ satisfy condition (8) . Moreover, for any $x \in B_{\alpha}$, $\alpha=a_{1} a_{2} \cdots a_{k}$,

(i) The point $x \tau_{i k}$-traces $u$ on $\left[T_{m(2 i-1)}, T_{m(2 i-1)+1}\right]$, and $\tau_{i k}$-traces $v$ on $\left[T_{m(2 i)}, T_{m(2 i)+1}\right]$, where $\tau_{i k}=\delta_{i+1}+\delta_{i+2}+\cdots+\delta_{k}<\delta_{i}, 1 \leq i<k$.

(ii) For any $i, x \tau_{i k}$-traces $a_{1}$ on any interval $\left[T_{m(2 i)+1}, T_{m(2 i)+2}\right]$ if $1 \leq i \leq k$, $\tau_{i k}$-traces $a_{2}$ on any interval $\left[T_{m(2 i)+2}, T_{m(2 i)+3}\right]$ with $2 \leq i \leq k$ and, in general, for any $j, 1 \leq j \leq k, x_{\alpha} \tau_{i k}$-traces $a_{j}$ on any $\left[T_{m(2 i)+j}, T_{m(2 i)+j+1}\right]$ with $j \leq i \leq k$.

To finish the argument put $S=\bigcap_{n=1}^{\infty} \bigcup_{\alpha \in\{u, v\}^{n}} B_{\alpha}$. Since $\operatorname{diam}\left(B_{\alpha}\right) \rightarrow 0$ for $n \rightarrow \infty, S \subset G$ is a Cantor set, by Lemma 1 . Moreover, any $s \in S$ can be uniquely represented as $s_{\alpha}=\bigcap_{n=1}^{\infty} B_{a_{1} a_{2} \cdots a_{n}}$, where $\alpha=a_{1} a_{2} \cdots a_{n} \cdots$ is a sequence in $\{u, v\}^{\mathbb{N}}$. Then $S$ is a scrambled set since $s_{\alpha} \delta_{i}$-traces $a_{j}$ on $\left[T_{m(2 i)+j}, T_{m(2 i)+j+1}\right]$, for any $j<i$. This implies (77).

Proof of Theorem 2. By Lemma 2, it is possible to define a sequence $S_{1}, S_{2}, \cdots$ of disjoint Cantor sets such that $S=\bigcup_{n=1}^{\infty} S_{n}$ is dense in $X$ and, for any distinct $x, y$ in $S$, (77) is satisfied. Let $\left\{G_{i}\right\}_{i=1}^{\infty}$ denote a countable base of topology of $X$ consisting of open balls. Apply Lemma 2 to $\nu=1,2,3, \ldots$, with $g$ and $\delta$ fixed to be the center and the radius of $G_{1}$, respectively. We get a Cantor $D 1$-scrambled set $S_{1}:=S$ satisfying (7), and a sequence $\mu:=\mu_{1}=m(1,1)<m(1,2)<m(1,3)<$ ... In the second step, if $S_{1} \neq X$, let $g \in X$ and $\delta>0$ be the center and the radius of the first open ball $G_{k}$ such that $G_{k} \cap S_{1}=\emptyset$. Apply Lemma 2 to $\nu=\{m(1,1), m(1,3), m(1,5), \cdots\}$ to obtain a set $S_{2}:=S$ and a sequence $\mu:=\mu_{2}=\{m(2,1), m(2,2), m(2,3), \cdots\} \subset \mu_{1}$. Then, for any $a \in S_{1}$ and $b \in S_{2}$, $a \delta_{i}$-traces $b$ on $\left[T_{m(2,2 i-1)}, T_{m(2,2 i-1)+1}\right]$, while $a \delta_{i}$-traces $u$ and $b \delta_{i}$-traces $v$ on $\left[T_{m(2,2 i)}, T_{m(2,2 i)+1}\right]$, for any $i \geq 1$; whence $S_{1} \cup S_{2}$ is a $D 1$-scrambled set, since also either $S_{1}$ or $S_{2}$ are $D 1$-scrambled sets. The result now follows by induction.

Notice that it never happens that $S_{1} \cup \ldots \cup S_{n}=X$ since a $D 1$-scrambled set is also $\varepsilon$-scrambled and such a set is never equal to the whole compactum [4].

Corollary 1. Every map $f \in C\left(I^{k}\right)$ with a weak specification property is conjugate to some map $g \in C\left(I^{k}\right)$ which exhibits DC1 a.e. 
Proof. The desired map $g$ is obtained by an application of Theorems 2 and 1 .

Remark 2. If there are points $u, v \in X$ such that $\lim _{n \rightarrow \infty} \rho\left(f^{n}(u), f^{n}(v)\right)=$ $\operatorname{diam} X$, then the $D 1$-scrambled set in Theorem 2 fulfills Definition 4 i.e. distributional chaos is extremal.

Remark 3. If $f$ has PSP, then it has WSP and a pair of distal points (e.g., formed by two distinct periodic points). In particular, the assumptions of Theorem 2 are fulfilled.

Let us recall the notion of Hausdorff dimension. Let $s>0$, and let $A$ be a subset of a compact metric space $(X, \rho)$. Denote by $\mathcal{B}_{n}$ the system of open balls in $X$ with diameter less than $1 / n$, and let $\mathcal{H}_{s, n}(A)=\inf _{\mathcal{G}} \Sigma_{G \in \mathcal{G}}|G|^{s}$, where the infimum is taken over all covers $\mathcal{G} \subset \mathcal{B}_{n}$ of $A$. Then $\mathcal{H}_{s}(A)=\lim _{n \rightarrow \infty} \mathcal{H}_{s, n}(A)$ is a nonincreasing function of $s$. The Hausdorff dimension $\operatorname{dim}(A)$ of $A$ is the infimum of $s$ such that $\mathcal{H}_{s}(A)<\infty$. Obviously, any set $A \subset I^{k}$ with $\operatorname{dim}(A)=0$ has zero Lebesgue measure. Let us continue with the following well-known lemma.

Lemma 3. There is a sequence $\left\{F_{n}\right\}_{n=1}^{\infty}$ of pairwise disjoint Cantor sets in I such that $\operatorname{dim}\left(I \backslash \bigcup_{n=1}^{\infty} F_{n}\right)=0$.

Proof. Let $\Lambda:=\left\{\lambda_{n}\right\}_{n=1}^{\infty}$, where $\lambda_{n}=1 / n^{n !}$. Let $F_{1}=I \backslash \bigcup_{n=1}^{\infty} I_{1, n}$, where $I_{1, n}$ are open intervals dense in $I$, with pairwise disjoint closures, such that $\left\{\left|I_{1, n}\right|\right\}_{n=1}^{\infty}$ is a subsequence of $\Lambda$. To get $F_{2}$, we let $I_{2, n}, n \in \mathbb{N}$, be open intervals in $I \backslash F_{1}$, with pairwise disjoint closures, and dense in any $I_{1, n}$ such that $\left\{\left|I_{2, n}\right|\right\}_{n=1}^{\infty}$ is a subsequence of $\Lambda$ and $\Sigma_{n=1}^{\infty}\left|I_{2, n}\right|^{1 / 2}<1$. In the next step we obtain open intervals $I_{3, n}, n \in \mathbb{N}$, with disjoint closures and dense in $I \backslash\left(F_{1} \cup F_{2}\right)$ such that $\Sigma_{n=1}^{\infty}\left|I_{3, n}\right|^{1 / 3}<$ 1, etc. Taking $S=F_{1} \cup F_{2} \cup \cdots$ we get $\mathcal{H}_{1 / n}(I \backslash S)<1$, for any $n>1$, whence $\operatorname{dim}(I \backslash S)=0$.

Corollary 2. Every bitransitive map $f \in \mathcal{C}(I)$ is conjugate to a map $g \in \mathcal{C}(I)$ which exhibits DC1 everywhere, except for a set of zero Hausdorff dimension. In particular, $g$ is DC1 a.e.

Proof. Map $f$ has SSP (see [5]). Hence the result follows by Theorem 2 and Lemma 3 via the fact that if $A, B \subset I$ are sets dense in $I$ and each of them is the union of pairwise disjoint Cantor sets, then there is an increasing homeomorphism $\varphi: I \rightarrow I$ such that $\varphi(A) \subseteq B$.

Conjecture 1. Corollary 2 cannot be extended to maps in $\mathrm{C}\left(I^{k}\right)$, with $k>0$.

Corollary 3. For any $k>0$ there exists a map $f \in C\left(I^{k}\right)$ which displays extremal DC1 a.e.

Proof. Let us define a piecewise linear map $g$ from the unit interval into itself with graph as presented on Figure1(i.e. $g(0)=0, g\left(\frac{1}{3}\right)=1, g\left(\frac{2}{3}\right)=0, g(1)=1$ ). Points 0,1 are fixed points of $g$. It is also easy to verify that $g$ is bitransitive; thus it has $S S P$.

Given a positive integer $k$ we define a map $\tilde{g} \in C\left(I^{k}\right)$ by the formula

$$
\tilde{g}\left(x_{1}, \ldots, x_{n}\right)=\left(g\left(x_{1}\right), \ldots, g\left(x_{n}\right)\right) .
$$

Map $\tilde{g}$ exhibits the strong specification property (it is a product of maps with $S S P$ [8]) and has two stationary points, $p_{0}=(0, \ldots, 0), p_{1}=(1, \ldots, 1)$. If $d$ denotes the 


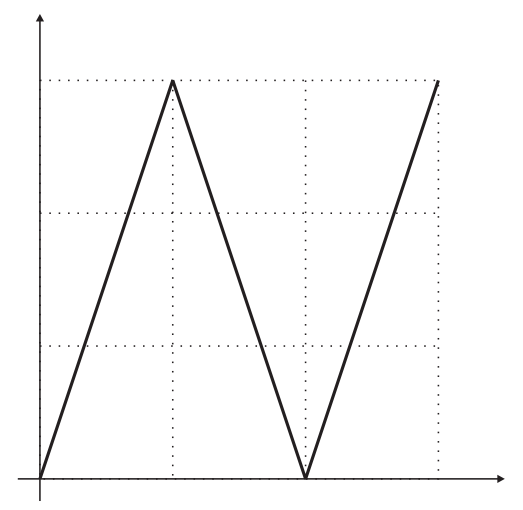

Figure 1. An interval map with extremal $D C 1$

Euclidean metric in $I^{k}$, then $d\left(p_{0}, p_{1}\right)=\operatorname{diam} I^{k}$. Let $f \in C\left(I^{k}\right)$ be a map obtained from $\tilde{g}$ by the application of the homeomorphism $h \in C\left(I^{k}\right)$ given by Theorem1(i.e. $\left.f=h \circ \tilde{g} \circ h^{-1}\right)$. Points $p_{0}, p_{1}$ are also fixed points of $f$ because $h$ is the identity on the boundary of $I^{k}$. Points $p_{0}, p_{1}$ were used in the construction of the $D 1$-scrambled set $S$ for $\tilde{g}$ by the technique described in Theorem 2 and by conjugacy arguments, similar properties are shared by $h(S)$ and $f$. In particular, $f$ exhibits extremal distributional chaos with a $D 1$-scrambled set $h(S)$ of full Lebesgue measure.

Given $f \in C(X)$ acting on a compact metric space and a point $x \in X$, we define an asymptotic cell of $x$ by the formula

$$
A(f, x)=\left\{y \in X: \lim _{n \rightarrow \infty} \rho\left(f^{n}(x), f^{n}(y)\right)=0\right\} .
$$

Theorem 3. Let us consider a map $f \in C\left(X_{0}\right)$ acting on a compact arcwiseconnected metric space $\left(X_{0}, \rho_{0}\right)$, with a weak specification property. Additionally, let us assume that $g_{i} \in C\left(X_{i}\right)$ are maps acting on compact metric spaces $\left(X_{i}, \rho_{i}\right)$ such that for any $i=1, \ldots, n$ there exists a point $z_{i} \in X_{i}$ such that $A\left(g_{i}, z_{i}\right)$ is dense in $X_{i}$. In that case the product map

$$
F=f \times g_{1} \times \ldots \times g_{n}: X_{0} \times \ldots \times X_{n} \longrightarrow X_{0} \times \ldots \times X_{n}
$$

has dense D1-scrambled set $S=\bigcup_{i=1}^{\infty} S_{i}$ where each $S_{i}$ is a Cantor set.

Lemma 4. If we additionally assume that $X$ is arcwise-connected, then the set $S$ from Theorem 2 may be decomposed into a countable sum of pairwise disjoint sets $P_{i}$ such that each $P_{i}$ is dense and is obtained as a countable sum of pairwise disjoint Cantor sets.

Proof. We perform a construction almost identical to that in the proof of Theorem 2. We start with some countable base $\left\{\tilde{B}_{i}\right\}_{i=1}^{\infty}$ of $X$ and rearrange it to obtain a base $\left\{B_{i}\right\}_{i=1}^{\infty}$ of the form

$$
\left\{B_{i}\right\}_{i=1}^{\infty}=\tilde{B}_{1}, \tilde{B}_{2}, \tilde{B}_{2}, \tilde{B}_{3}, \tilde{B}_{3}, \tilde{B}_{3}, \tilde{B}_{4}, \ldots
$$

We repeat the construction performed in the proof of Theorem 2, with the only addition that the open ball $B_{k}$ chosen in step $n$ has not only the property that

$$
B_{k} \cap\left(S_{1} \cup \ldots \cup S_{n}\right)=\emptyset
$$


but additionally $B_{k} \subset B_{n}$. That this is possible follows directly from the definition of an arcwise-connected space: There always exists an $\operatorname{arc} \alpha: I \rightarrow X$ such that $\alpha(I) \subset B_{n}$. But then, $\alpha(I) \backslash\left(S_{1} \cup \ldots \cup S_{n}\right) \neq \emptyset$, as otherwise $I$ would not be connected. This implies that $B_{n} \backslash\left(S_{1} \cup \ldots \cup S_{n}\right) \neq \emptyset$; in particular, this complement contains some $B_{k}$ as a subset.

By construction, each $B_{n}$ contains some Cantor set constructed inside of it in the step $n$. We may rewrite the base $\left\{B_{k}\right\}_{k=1}^{\infty}$ in the form $\left\{B_{k}\right\}_{k=1}^{\infty}=\left\{C_{i, j}\right\}_{1 \leq i \leq j}$, where $C_{i, j}=\tilde{B}_{j}$. In the same way we may change indices of $\left\{S_{k}\right\}_{k=1}^{\infty}$ to the form $S_{i, j}$ and then $S_{i, j} \subset C_{i, j}$.

Given an integer $i$ we define

$$
P_{i}=\bigcup_{j=1}^{\infty} S_{i, j} .
$$

Observe that $P_{i} \cap P_{k}=\emptyset$ for $i \neq k$. Furthermore, each $P_{i}$ is dense in $X$ by the fact that for any fixed $i$ we have the equality

$$
\left\{C_{i, j}\right\}_{j=i}^{\infty}=\tilde{B}_{i}, \tilde{B}_{i+1}, \tilde{B}_{i+2}, \tilde{B}_{i+3}, \ldots ;
$$

in particular $\left\{C_{i, j}\right\}_{j=i}^{\infty}$ is a base of the topology of $X$.

Proof of Theorem 3. Let us denote by $d$ the product metric

$$
d\left(\left(x_{1}, \ldots, x_{n}\right),\left(y_{1}, \ldots, y_{n}\right)\right)=\max _{i=1, \ldots, n} \rho_{i}\left(x_{i}, y_{i}\right) .
$$

Let $x_{i}, y_{i} \in A\left(g_{i}, z_{i}\right)$. In that case, it is easy to verify that for any $x_{0}, y_{0} \in X_{0}$, $t>0$ and large $n$ it follows that

$$
\Phi_{\left(x_{0}, \ldots, x_{n}\right),\left(y_{0}, \ldots, y_{n}\right)}^{(n)}(F, t) \leq \Phi_{x_{0} y_{0}}^{(n)}(f, t) \leq \Phi_{\left(x_{0}, \ldots, x_{n}\right),\left(y_{0}, \ldots, y_{n}\right)}^{(n)}(F, 2 t) .
$$

In particular, when $\left(x_{0}, y_{0}\right)$ is a $D C 1$ pair, then so is the pair $\left(\left(x_{0}, \ldots, x_{n}\right)\right.$, $\left.\left(y_{0}, \ldots, y_{n}\right)\right)$.

There exists an at most countable and dense (in $X_{1} \times \ldots \times X_{n}$ ) set

$$
C=\left\{c_{1}, c_{2}, \ldots\right\} \subset \prod_{i=1}^{n} A\left(g_{i}, z_{i}\right) .
$$

Let $P_{i}$ be sets obtained by the application of Lemma 4 to the dynamical system $\left(f, X_{0}\right)$. The following set is the desired $D 1$-scrambled set:

$$
S=\bigcup_{i=1}^{\infty} P_{i} \times\left\{c_{i}\right\} .
$$

The above theorem obviously implies that there exists a nontransitive map on $I^{k}$ which displays uniform $D C 1$ a.e. for $k>1$. The following example shows that there is such a map for $k=1$, too.

Example 4. Let us consider a map $f:[0,1] \rightarrow[0,1]$ from Figure 2. The map $\left.f\right|_{[0,0.5]}$ has $S S P$, so we may apply Lemma 4 . In particular, there exist two dense (in $[0,0.5])$ and disjoint sets $S_{1}, S_{2} \subset(0,0.5)$ such that $S_{1} \cup S_{2}$ is a $D 1$-scrambled set for $f$. Observe that $f(1-x)=f(x)$ for any $x \in[0,0.5]$. This immediately implies that the set

$$
S=S_{1} \cup\left\{1-x: x \in S_{2}\right\}
$$

is a dense (in $[0,1]$ ) $D 1$-scrambled set for $f$. Applying Theorem 1 we obtain a nontransitive interval map which exhibits distributional chaos almost everywhere. 


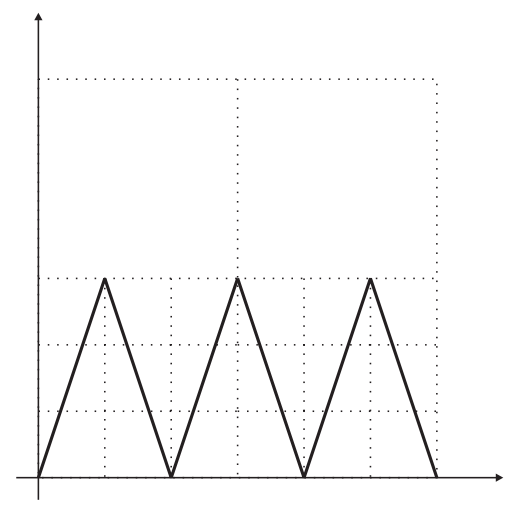

FIgURE 2. A map without $W S P$ which has a dense D1-scrambled set

\section{AcKnowledgements}

The main part of this paper was written during the first author's visit to the Mathematical Institute of the Silesian University in Opava. This research was supported by project MSM4781305904 from the Czech Ministry of Education. The first author was supported by Polish Ministry of Science and Higher Education grant No. NN201272333 for years 2007-2009, an annual national scholarship for young scientists from the Foundation for Polish Science, and AGH grant No. 10.420.03. The second author was supported by project 201/06/0318 from the Czech Science Foundation.

\section{REFERENCES}

[1] M. Babilonová, The bitransitive continuous maps of the interval are conjugate to maps extremely chaotic a.e., Acta Math. Univ. Comen. 69 (2000) (2), 229-232. MR.1819523 (2002f:37062)

[2] M. Babilonová-Štefánková, Extreme chaos and transitivity, Internat. J. Bifur. Chaos Appl. Sci. Engrg. 13 (2003), 1695-1700. MR2015619 (2004i:37075)

[3] W. Bauer and K. Sigmund, Topological dynamics of transformations induced on the space of probability measures, Monatsh. Math. 79 (1975), 81-92. MR0370540 (51:6767) $\mathrm{W}$

[4] F. Blanchard, W. Huang and L. Snoha, Topological size of scrambled sets, Colloquium Math. 110 (2008) 293-361. MR2353910

[5] A. M. Blokh, On graph-realizable sets of periods, J. Difference Equ. Appl. 9 (2003), 343-357. MR.1990341 (2004j:37072)

[6] R. Bowen, Topological entropy and axiom A, in "Global Analysis", Proceedings of Symposia on Pure Mathematics, vol. 14, Amer. Math. Soc., Providence, RI, 1970. MR0262459 (41:7066)

[7] A. M. Bruckner and T. Hu, On scrambled sets for chaotic functions, Trans. Amer. Math. Soc. 301 (1987), 289-297. MR879574 (88f:26003)

[8] M. Denker, C. Grillenberger and K. Sigmund, Ergodic theory on compact spaces, SpringerVerlag, Berlin, 1976. MR0457675 (56:15879)

[9] W. J. Gorman III, The homeomorphic transformation of c-sets into d-sets, Proc. Amer. Math. Soc. 17 (1966), 825-830. MR0207921 (34:7734)

[10] W. Huang and X. Ye, Homeomorphisms with the whole compacta being scrambled sets, Ergod. Th. Dynam. Sys. 21 (2001), 77-91. MR.1826661(2002d:37020)

[11] I. Kan, A chaotic function possessing a scrambled set with positive Lebesgue measure, Proc. Amer. Math. Soc. 92 (1984), 45-49. MR749887 (86b:26009a) 
[12] K. Kuratowski, Topology, Vol. II, Academic Press and Polish Scientific Publishers, 1968. MR0259835(41:4467)

[13] G. F. Liao, L. D. Wang, X. D. Duan, A chaotic function with a distributively scrambled set of full Lebesgue measure, Nonlinear Analysis - Theory, Methods \& Applications 66 (2007), 2274-2280. MR2311031(2008c:37020)

[14] M. Misiurewicz, Chaos almost everywhere, in: "Iteration Theory and Its Functional Equations" (ed., G. Targonski et al.), Lecture Notes in Mathematics 1163, 125-130, Springer, Berlin, 1985. MR829765 (87e:58152)

[15] J. C. Oxtoby and S. M. Ulam, Measure-preserving homeomorphisms and metrical transitivity, Ann. of Math. 42 (1941), 874-920. MR0005803(3:211b)

[16] B. Schweizer and J. Smítal, Measures of chaos and a spectral decomposition of dynamical systems on the interval, Trans. Amer. Math. Soc. 344 (1994), 737-854. MR1227094 (94k:58091)

[17] K. Sigmund, On dynamical systems with the specification property, Trans. Amer. Math. Soc. 190 (1974), 285-299. MR0352411 (50:4898)

[18] J. Smítal, A chaotic function with some extremal properties, Proc. Amer. Math. Soc. 87 (1983), 54-56. MR677230 (84h:26008)

[19] J. Smítal, A chaotic function with a scrambled set of positive Lebesgue measure, Proc. Amer. Math. Soc. 92 (1984), 50-54. MR749888(86b:26009b)

Faculty of Applied Mathematics, AGH University of Science and Technology, al. Mickiewicza 30, 30-059 Kraków, Poland

E-mail address: oprocha@agh.edu.pl

Mathematical Institute, Silesian University, 74601 Opava, Czech Republid

E-mail address: marta.stefankova@math.slu.cz 